\title{
Panorama sobe-desce e varal do SUS: o que motiva e o que desmotiva a utilização do Sistema Único de Saúde?
}

Panorama Up - Down and Clothesline of SUS: what motivates and what discourages the use of the Sistema Único de Saúde (health system)?

Panorama arriba-abajo y varal del SUS: ¿lo que estimula y lo que desestimula a la utilización del Sistema Único de Salud?

Recebido: 8 jan 2017 Revisado: 22 jan 2018 Aceito: 23 jan 2018

\section{Autor de}

correspondência:

Rosângela Minardi Mitre Cotta

rosangelaminardi@gmail. com

Conflito de interesses: Os autores declaram não haver nenhum interesse profissional ou pessoal que possa gerar conflito de interesses em relação a este manuscrito.

Financiamento: Projeto CAPES $n$. 23038.009788/2010-78
(1) Universidade Federal de Viçosa - UFV, Departamento de Nutrição e Saúde, Viçosa, MG, Brasil.

(2) Universidade Federal do Sul e Sudeste do Pará - UNIFESSPA, Faculdade de Saúde Coletiva, Marabá, PA, Brasil.

(3) Universidade Federal de Uberlândia - UFU, Faculdade de Medicina, Uberlândia, MG, Brasil.

(4) Universidade Federal Fluminense - UFF, Faculdade de Medicina, Niterói, RJ, Brasil.

(5) Faculdade de Medicina de Barbacena - FUNJOBE, Barbacena, MG, Brasil.

(6) Universidade Federal do Rio de Janeiro - UFRJ, Rio de Janeiro, RJ, Brasil.

(7) Universidade do Estado do Rio de Janeiro - UERJ, Rio de Janeiro, RJ, Brasil.

\section{Resumo}

A percepção dos usuários acerca dos serviços prestados em saúde tem sido evidenciada na literatura científica como importante estratégia para se avaliar a qualidade destes serviços oferecidos à população. Neste sentido, o presente estudo tem como objetivo avaliar os fatores motivadores e desmotivadores da utilização do Sistema único de Saúde (SUS) e as experiências positivas e negativas vivenciadas pelos usuários do sistema de saúde. Trata-se de pesquisa qualitativa que utilizou como método, o estudo de caso, tendo como referência o Panorama Sobe-Desce e o Varal do SUS - técnicas criativas e interativas de investigação em saúde. Entre os fatores que motivam a utilização dos SUS, destacam-se: a acessibilidade; os serviços de alta complexidade; a qualidade; a universalidade; o atendimento integral e o acesso a especialistas. Como aspectos desmotivadores destacam-se: a falta de profissionais, a organização dos serviços deficiente, as filas de espera, a infraestrutura precária, a falta de humanização e o desconhecimento em relação ao SUS. A realização de estudos que levem em conta a percepção dos usuários sobre a qualidade do cuidado é importante para o planejamento e melhoria das políticas de saúde.

Descritores: Serviços de Saúde; Sistema Único de Saúde; Percepção; Política de Saúde. 


\begin{abstract}
The perception of users about health services has been evidenced in the scientific literature as an important strategy to evaluate the quality of these services offered to the population. In this sense, the present study has as objective to evaluate the motivating and demotivating factors of the use of the Sistema Único de Saúde (SUS) and the positive and negative experiences experienced by the users of the health system. It is a qualitative research that used as a method, the case study, having as reference the Panorama Up - Down and the Clothesline of SUS - creative and interactive techniques of health research. Among the factors that motivate the use of SUS, the following stand out: accessibility; high complexity services; the quality; the universality; the integral care and the access to specialists. The lack of motivation include the lack of professionals, poor service organization, waiting lines, poor infrastructure, lack of humanization and lack of knowledge about SUS. The realization of studies that take into account the perception of the users about the quality of care is important for the planning and improvement of health policies.
\end{abstract}

Keywords: Health Care; Health System; Perception; Health Policy.

\begin{abstract}
Resumen
La percepción de los usuarios acerca de los servicios de salud se ha demostrado en la literatura científica como una estrategia importante para evaluar la calidad de los servicios ofrecidos a la población. En este sentido, el presente estudio tiene como objetivo evaluar los factores de motivación y desmotivación del uso del Sistema Único de Saúde (SUS) y de las experiencias positivas y negativas de los usuarios del sistema de salud. Se trata de una investigación cualitativa que utiliza el método, el estudio de caso, con referencia al Panorama arriba-abajo y Varal del SUS - técnicas creativas e interactivas de la investigación en salud. Entre los factores que motivan el uso de SUS, son: la accesibilidad; los servicios de alta complejidad; la calidad; universalidad; atención integral y acceso a especialistas. Como aspectos de desmotivación destacan: la falta de profesionales, la organización de servicios de baja calidad, las listas de espera, la infraestructura deficiente, la falta de humanización y la ignorancia sobre el SUS. Los estudios que tengan en cuenta la percepción de la calidad de la atención de los usuarios es importante para la planificación y la mejora de las políticas de salud.
\end{abstract}

Palabras-claves: Servicios de Salud; Sistema de Salud, Percepción, Política de Salud.

\title{
Introdução
}

O Sistema Único de Saúde - SUS surgiu a partir de um histórico processo de lutas do movimento sanitário brasileiro, tendo como princípios a universalidade, integralidade, equidade, descentralização, hierarquização e regionalização e participação social. ${ }^{1}$

Legalmente, a proposta do SUS foi incorporada à Carta Constitucional de 1988 e com isso, a saúde torna-se direito de todos e dever do Estado, garantida mediante políticas sociais e econômicas que visem à redução do risco de doenças e de outros agravos e ao acesso universal e igualitário às ações e serviços para sua promoção, proteção e recuperação. $^{2}$

Assim, sendo a população em sua totalidade beneficiária das ações em saúde, a 
opinião dos usuários quanto à qualidade da atenção é considerada de extrema relevância quando se busca avaliar esses serviços. Neste sentido, a percepção dos usuários acerca dos serviços prestados em saúde tem sido evidenciada na literatura científica como importante estratégia para se avaliar a qualidade destes serviços oferecidos à população. 3,4,5 Para tanto, diversos fatores devem ser analisados, como a qualidade do atendimento oferecido, a garantia do acesso às ações e serviços de saúde, a estrutura física e organizacional, a relação profissional de saúde-usuário, o acolhimento e o vínculo. ${ }^{1,6,7}$

Estudos sobre a percepção dos usuários em relação ao funcionamento e às funções e (dis)funções do SUS podem contribuir para a melhoria do sistema sanitário, servindo de parâmetro para o planejamento, ações e políticas de saúde. ${ }^{8}$ Assim, a mensuração da satisfação é um instrumento importante para a administração e planejamento, além de desempenhar um papel importante entre o prestador de serviços e o usuário, pois reflete no julgamento da qualidade dos cuidados prestados. ${ }^{9}$

A participação dos usuários, quando ocorrida de forma livre e crítica, pode contribuir para o empoderamento/libertação deste grupo populacional, questões estas essenciais para a viabilização de políticas de promoção da saúde e prevenção de agravos e controle de enfermidades. Desse modo, a melhoria dos programas de saúde pode ser baseada no processo de avaliação apoiado na análise do sistema por meio da ótica do usuário, servindo de parâmetro para as ações e políticas de saúde. ${ }^{10}$

Neste sentido, o presente estudo teve por objetivo compreender os fatores motivadores e desmotivadores da utilização do SUS, evidenciando as experiências positivas e negativas em relação a estes serviços.

\section{Metodologia}

Os dados foram coletados durante o Simpósio de Integração Acadêmica - SIA da Universidade Federal de Viçosa - UFV. O público alvo foram os membros da comunidade 
acadêmica da UFV que visitaram o SIA durante uma semana no mês de dezembro de 2012 e aceitaram participar da pesquisa.

Trata-se de um estudo quanti-qualitativo, o qual utilizou como método o estudo de caso, modalidade empírica empregada de investigação quando não é possível diferenciar o fenômeno estudado de seu contexto, e pretende-se buscar um conhecimento circunstanciado a respeito de um tema ${ }^{11}$, no caso deste trabalho, as experiências vivenciadas pelos brasileiros acerca do SUS. Como instrumento de coleta de dados, estes foram obtidos por meio de duas técnicas: o Panorama Sobe-Desce e o Varal do SUS.

O Panorama Sobe-Desce é uma técnica que tem como princípio a expressão dos participantes sobre suas motivações (Sobe) e desmotivações (Desce) em determinado estudo, curso, programa ou estratégia, de forma lúdica, interativa e criativa. As respostas a uma determinada pergunta formulada pelos pesquisadores são respondidas pelos participantes em post its de duas cores distintas: uma para cada percepção - motivação e desmotivação que colados em um banner pelos próprios respondentes formam um panorama onde o contexto de cada um em particular e do grupo como um todo é evidenciado. Este recurso educacional foi desenvolvido pelas autoras Denise Herdy Afonso e Lia Márcia Cruz da Silveira e encontrase publicado no Caderno de Ensinagem do Tutor, material de uso específico da Equipe Docente do Curso de Desenvolvimento de Competências Pedagógicas para prática da Preceptoria e Docência.

Neste estudo, trabalhou-se com a seguinte pergunta: "Em sua opinião, quais fatores que motivam (Sobe) e que desmotivam (Desce) a utilização do SUS?". As respostas foram relatadas em post its de cores azul (sobe) e branco (desce) e afixadas no cartaz pelos próprios participantes (Figura 1). 


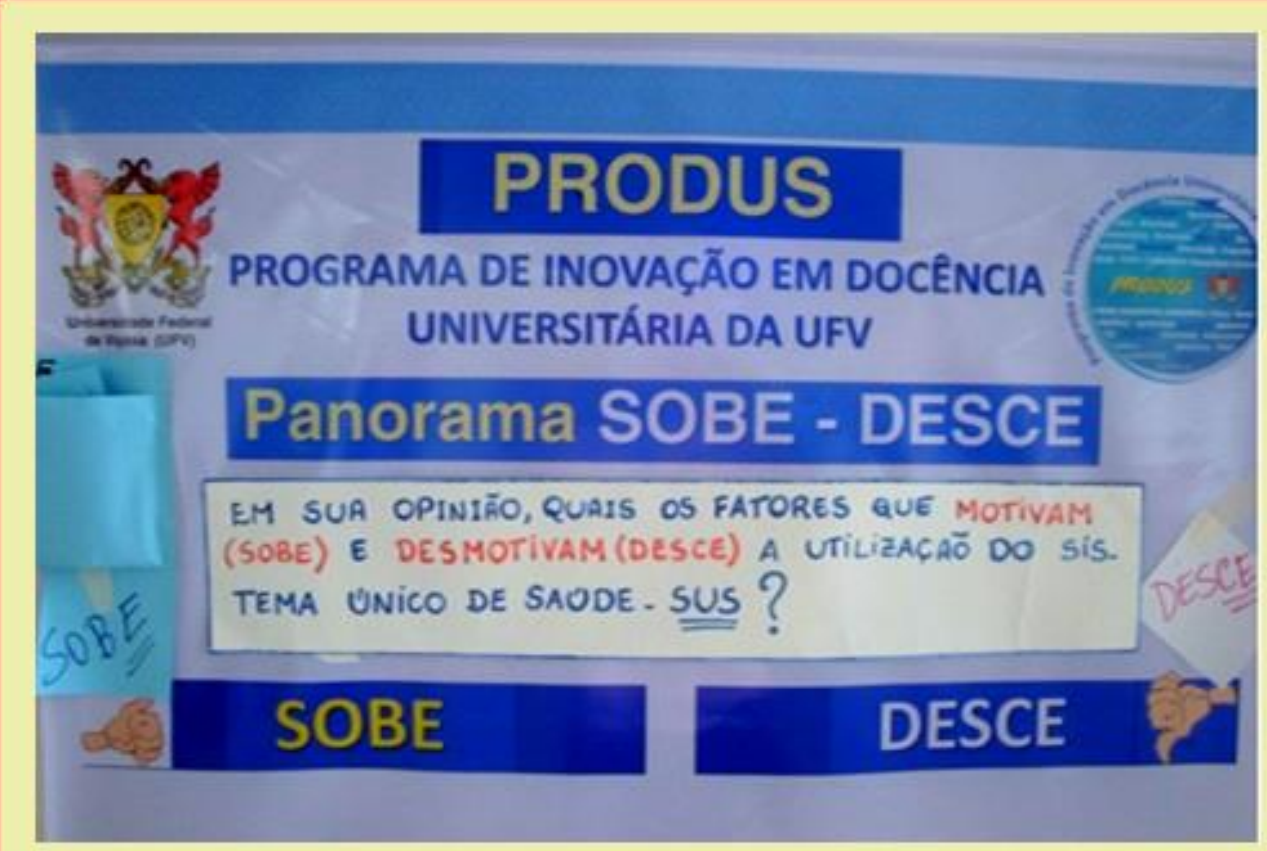

Figura 1. Panorama Sobe-Desce das motivações e desmotivações de utilização do SUS Fonte: Elaboração própria (2012).

Por sua vez, o Varal do SUS, é uma técnica que visa captar o relato dos usuários sobre suas experiências positivas e negativas em relação à utilização dos serviços do SUS. Trata-se de um painel, onde os participantes responderam a seguinte pergunta: "Você já foi atendido em alguma unidade de saúde em qualquer momento da sua vida pelo SUS? Conte aqui a sua experiência como usuário". As respostas foram relatadas em post its de cores azul (experiência positiva) e branco (experiência negativa), e afixadas, pelos próprios usuários, em um varal, com auxílio de prendedores (Figura 2). 


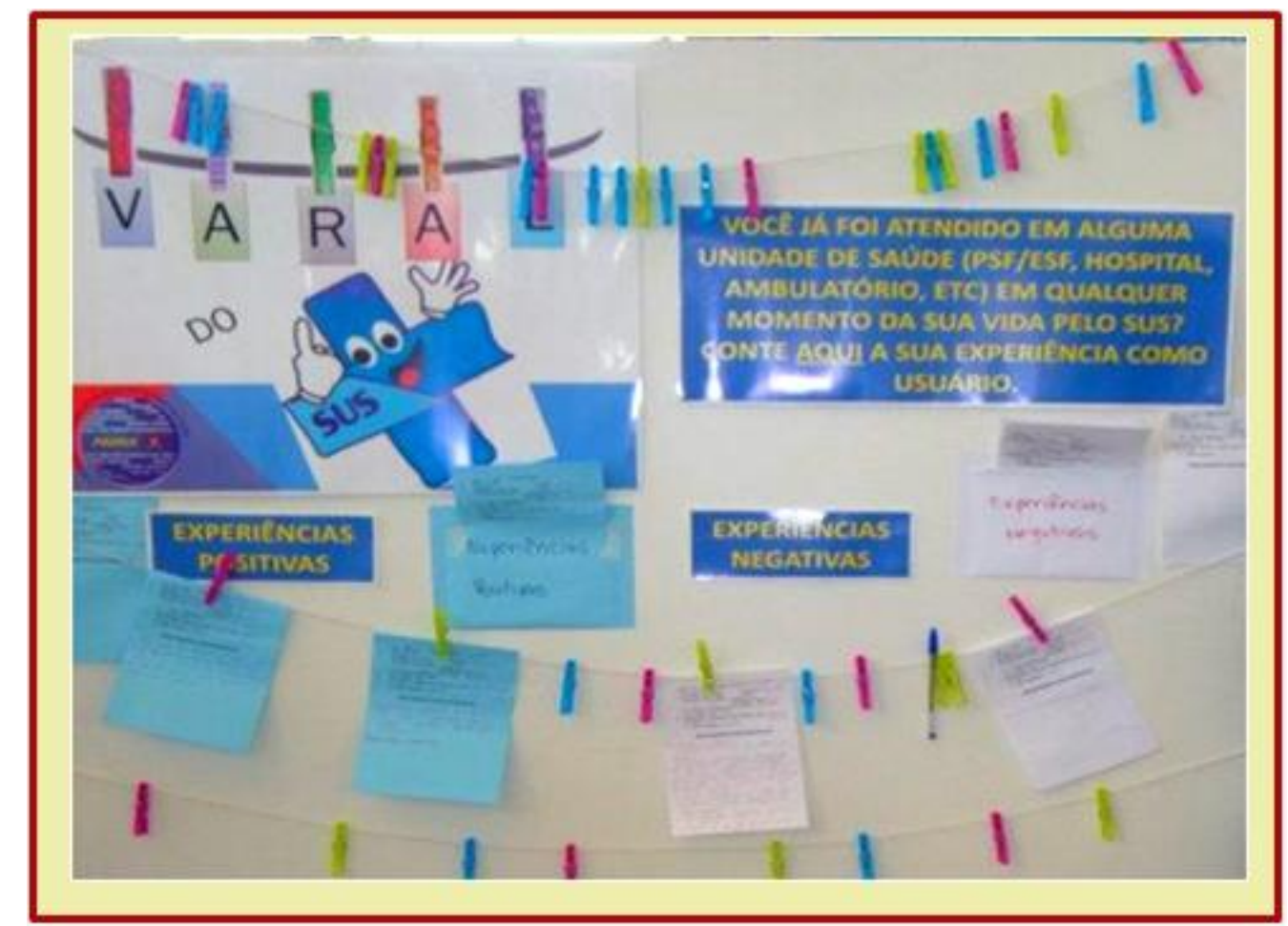

Figura 2. Varal do SUS sobre as experiências positivas e negativas dos usuários Fonte: Elaboração própria (2012).

Os dados quantitativos foram analisados através do cálculo da frequência das variáveis. Com relação aos dados qualitativos, após leitura global e exaustiva das respostas dos participantes, foi realizada a Análise de Conteúdo cumprindo três etapas: (1) Pré-análise; (2) Exploração do material; e (3) Tratamento dos resultados, inferência e interpretação ${ }^{12}$, o que permitiu identificar os núcleos de sentido e organizar as categorias sobre os fatores motivadores e desmotivadores em relação à utilização do SUS.

\section{Resultados}

Responderam às perguntas do Panorama Sobe-Desce 47 pessoas e do Varal do SUS 45 pessoas. Do total dos participantes, 57,4\% era do sexo feminino, tinham idade entre 19 e 64 anos e média de 26,2 anos. Quanto à ocupação, 78,7\% era composta por estudantes da 
graduação $(78,7 \%), 6,4 \%$ por estudantes da pós-graduação e 12,8\% por técnicos administrativos.

Segundo a análise do Panorama Sobe-Desce, dentre os fatores que motivaram a utilização dos SUS, 78,94\% dos participantes citaram a acessibilidade como ponto forte do SUS, propiciada pela gratuidade do sistema de saúde quanto ao acesso a atendimentos, exames, vacinas e medicamentos. Outros fatores motivadores também citados foram: disponibilidade de serviços de alta complexidade com qualidade (21,05\%); a universalidade (21,05\%); acesso a especialistas (10,52\%), tais como: oncologistas, hematologistas (Quadro 1).

Dentre os fatores que desmotivaram a utilização do SUS, 50\% dos participantes citaram a falta de profissionais, destacando o número reduzido e a falta de capacitação destes; $50 \%$ também citaram a demora no atendimento, muitas vezes relacionada à falta de profissionais e à grande demanda, fazendo com que haja sobrecarga de trabalho e consequente mau atendimento, citado por 26,92\% dos participantes, além de relatarem a formação de filas, aspecto observado em $38,46 \%$ dos relatos.

\section{Aspectos motivadores (Sobe)}

Acessibilidade, proporcionada pela gratuidade do sistema de saúde Disponibilidade de serviços de Alta Complexidade Atendimento de qualidade

Universalidade Atendimento integral

Acesso a especialistas

\section{Aspectos desmotivadores (Desce)}

Falta de compromisso (número reduzido e falta de capacitação dos profissionais)

Demora no atendimento (falta de profissionais e grande demanda)

Desconhecimento da população em relação ao SUS

Ausência de cuidado humanizado e integral

Espera em filas

Quadro 1. Fatores motivadores (sobe) e desmotivadores (desce) em relação à utilização do SUS, Viçosa, Minas Gerais, 2012.

Fonte: Elaboração própria (2012). 
Outros fatores desmotivadores foram: a falta de estrutura das Unidades de Saúde (38,46\%); falta de recursos públicos $(7,69 \%)$, falta de acesso a determinadas especialidades médicas (7,69\%); ausência de cuidado humanizado e integral (7,69\%); desorganização e descaso por parte do governo (3,84\%) e desconhecimento da população em relação ao SUS (3,84\%) (Quadro 1).

Em relação ao Varal do SUS, houve a participação de 47 pessoas. Quanto à profissão, todos eram vinculados à UFV, com exceção de uma pessoa, que era advogada. As profissões vinculadas à UFV se dividiram em: estudante da graduação $(78,7 \%)$, estudante da pósgraduação (6,4\%) e técnicos administrativos (12,8\%).

A análise do Varal do SUS, por sua vez, mostrou semelhanças quanto às experiências positivas $(n=23)$ e negativas $(n=24)$ relatadas pelos participantes, cujos alguns depoimentos serão apresentados a seguir (Quadro 2).

\section{Experiências positivas}

Como experiência positiva, posso citar a realização de uma cirurgia da mama para retirada de um nódulo.

Diversos exames de alto custo e complexidade. Recebi todos os medicamentos necessários para um tratamento que fiz durante 6 meses.

Realizei uma cirurgia cardíaca. Primeiro entrei na fila do SUS à espera de uma vaga. Em menos de 1 semana a vaga surgiu. Internei, tive um atendimento humanizado tanto pelos enfermeiros, médicos e outros funcionários. Estiveram disponíveis equipamentos de alta complexidade e recursos necessários para minha recuperação.

Realização de consulta para a saúde da mulher com a enfermeira da Unidade, encaminhamento ao médico devido a alteração. Acompanhamento domiciliar pelo Agente Comunitário de Saúde. Percebi a relação multiprofissional e a integralidade das ações.

\section{Experiências negativas}

Enquanto eu esperava atendimento na sala de espera, havia pessoas em estado crítico esperando serem chamadas e não havia priorização de atendimento. Com isso, um senhor de aproximadamente 70 anos, faleceu enquanto esperava atendimento

O hospital estava em obras na época. Péssimo atendimento realizado pelos técnicos em enfermagem, que esqueceram uma seringa de injeção em meu braço e fiquei esquecido no período noturno de internação.

Minha filha quebrou o braço e (na cidade) só é possível fazer gesso na rede do SUS. Precisei de 9 horas de espera para engessar o braço de uma criança de 4 anos. Foram 9 horas a partir do diagnóstico.

Quadro 2. Varal do SUS: relatos dos usuários acerca das experiências positivas e negativas de utilização do SUS, Viçosa, Minas Gerais, 2012.

Fonte: Elaboração própria (2012). 


\section{Discussão}

A percepção dos usuários acerca dos serviços prestados em saúde tem sido evidenciada na literatura científica como importante estratégia para se avaliar a qualidade destes serviços oferecidos à população. ${ }^{3,4,5,26}$ Neste contexto, o desenvolvimento de um sistema de avaliação de satisfação pode representar uma importante ferramenta para o desenvolvimento de estratégias de gestão para o setor de serviços. ${ }^{13}$ Para tanto, diversos fatores devem ser analisados, como a qualidade do atendimento oferecido aos usuários, a garantia do acesso às ações e serviços de saúde, a estrutura física e organizacional, a relação profissional de saúdeusuário, o acolhimento e o vínculo. $1,6,7,14$

Neste sentido, compreender os aspectos motivadores e desmotivadores em relação à utilização do SUS, evidenciando as experiências positivas e negativas dos usuários configuramse primordiais para o direcionamento das ações e cuidados em saúde. Assim como encontrado no presente estudo, a evidência científica destaca a importância da acessibilidade e da universalidade das ações. Estudo realizado por Martins et al., ${ }^{1}$ objetivando analisar as representações sociais do SUS e da Estratégia Saúde da Família - ESF pelos usuários, identificou que $48,5 \%$ dos usuários discursaram positivamente em relação ao serviço utilizado, no que se refere à gratuidade dos serviços. Ainda, segundo os mesmos autores, apenas 9,6\% tinham a concepção do SUS como um sistema universal, resultados inferiores ao encontrado no presente estudo, uma vez que a acessibilidade, propiciada pela gratuidade dos serviços, foi mencionada por $78,8 \%$ dos usuários e a universalidade das ações foi observada em $21,05 \%$ das respostas. Tal resultado corrobora com o Sistema de Indicadores de Percepção Social, que encontrou como principal ponto positivo do SUS, o acesso gratuito aos serviços de saúde prestados pelo sistema $(52,7 \%)$, seguido, pelo atendimento universal $(48,0 \%) .{ }^{15} \mathrm{O}$ acesso às ações e serviços de saúde é primordial para a efetiva implementação destes serviços, uma vez que as experiências vivenciadas pelos usuários do SUS refletem na quase totalidade dos casos, a capacidade do sistema em corresponder às suas expectativas e necessidades, ${ }^{16,17}$ garantindo- 
Ihes a saúde como direito inerente à todo e qualquer cidadão brasileiro, previsto pelo Constituição Federal. ${ }^{2}$

Ainda, em relação aos fatores motivadores dos usuários em relação aos serviços dos SUS, a distribuição gratuita de medicamentos, garantida à população por meio da Política Nacional de Medicamentos, ${ }^{8}$ foi relatada como experiência positiva pelos participantes do presente estudo (Quadro 1). Estudo realizado por Gouveia et al., ${ }^{5}$ com o objetivo de conhecer os fatores associados à satisfação dos usuários do sistema de saúde de Pernambuco, 56,6\% dos entrevistados relataram estarem satisfeitos com a disponibilidade de medicamentos pelo SUS. Para Souza, ${ }^{19}$ o SUS tem promovido o acesso aos medicamentos de forma equitativa, em especial àqueles de uso contínuo, como o fornecimento de anti-hipertensivos arteriais. Apesar da relevância do SUS no provimento de medicamentos, Boing et al., ${ }^{20}$ objetivando descrever a prevalência e os fatores associados ao acesso a medicamentos no SUS, que tiveram prescrição médica pelo próprio sistema, encontraram que apenas $45,3 \%$ dos obtiveram integralmente os medicamentos pelos SUS.

A integralidade das ações também foi mencionada como fator motivador da utilização do SUS (Quadro 1). Garantir a integralidade aos usuários é oferecer atendimento desde a Atenção Primária à Saúde - tendo aqui as equipes da ESF papel primordial no sucesso dessas ações - passando pelos encaminhamentos e atendimentos de serviços de média e alta complexidade quando necessitar. ${ }^{21}$ Estudo realizado por Gomes et al., ${ }^{14}$ em município de pequeno porte da zona da mata de Minas Gerais, 89,2\% dos entrevistados ressaltaram a importância da integralidade das ações para a resolutividade dos serviços oferecidos pelo SUS, especialmente pela realização das visitas domiciliares como parte das atividades desenvolvidas pelos Agentes Comunitários de Saúde - ACS, mencionadas pelos usuários como fundamentais para a melhora da qualidade de vida. Estes achados vão ao encontro do presente estudo, uma vez que o acompanhamento das famílias pelos ACS foi considerado experiência positiva da utilização dos serviços do SUS (Quadro 2).

Assim como os fatores motivadores e as experiências positivas, os participantes do 
presente estudo relataram as suas experiências negativas e o que os desmotivavam a utilizar os serviços do SUS. Os achados indicam uma predominância de percepções negativas do sistema de saúde, talvez porque estas pessoas só consigam vincular o SUS à assistência hospitalar, com forte influência do modelo biomédico; desconsiderando o papel importante das políticas públicas nas ações de promoção da saúde e prevenção de agravos e enfermidades. ${ }^{14}$ Desde esta perspectiva, destaca-se também a influência exercida pelos meios de comunicação, uma vez que as principais imagens e informações divulgadas pela mídia sobre o SUS são mais comumente associadas às mazelas e dificuldades do setor, quase sempre a partir de uma suposta ineficiência do Estado, incompetência das autoridades ou dos profissionais da área. ${ }^{22}$ Martins et al., ${ }^{1}$ reiteram que a visão focalizada do SUS representada pela população é o retrato da prática de implantação do sistema sanitário, segundo a qual foi alvo das tensões provocadas pelas "externalidades" advindas dos ajustes econômicos e políticos no Brasil. Entretanto, mesmo diante disto, não se pode deixar de destacar o notório avanço legal representado pela criação do SUS no Brasil, principalmente quando se pensa nos direitos sociais conquistados pela população. ${ }^{1}$

A demora no atendimento e as filas de espera também aparecem como um problema (Quadros 1 e 2). Estudo de Moimaz ${ }^{8}$ ilustra bem as possíveis causas das filas, onde se destaca que na trajetória da implantação de serviços de Atenção Primária à Saúde no Brasil, ao invés de se propiciar a integração entre as ações curativas, de prevenção e promoção, o que se assiste são filas de espera por atendimentos especializados. Ademais, são necessários maiores investimentos na infraestrutura e na capacitação dos profissionais para atuarem no SUS.

Assim como evidenciado no presente estudo, a relação entre o profissional de saúde e usuário é relatado na evidência científica, onde o desempenho profissional é avaliado pelo usuário por meio do interesse demonstrado através do exame físico, das orientações realizadas, bem como na resolutividade das condutas. ${ }^{8,23}$ A percepção dos usuários tem sido cada vez mais utilizada como uma medida importante na avaliação da qualidade dos cuidados em saúde. Para Gouveia, ${ }^{5}$ a percepção dos cuidados é influenciada pela expectativa do usuário, 
bem como pelos cuidados recebidos. O atendimento ao usuário, pautado na humanização enquanto estratégia de intervenção nos processos de trabalho vai ao encontro dos princípios do SUS, por propiciar o estabelecimento do vínculo entre usuário-serviço de saúde. Esse vínculo otimiza o processo da assistência, permitindo que os profissionais conheçam seus pacientes e as prioridades de cada um, facilitando-Ihes o acesso. ${ }^{24,25}$

Destarte, ouvir as satisfações, opiniões, sentimentos e inquietudes do usuário em relação ao serviço a ele oferecido tem sido valorizado por constituir-se um importante indicador da qualidade do serviço prestado. ${ }^{8}$

\section{Considerações finais}

O presente estudo apresentou de uma forma geral, uma avaliação positiva do SUS, entretanto, os diversos aspectos do atendimento, como organização dos serviços e da demanda, infraestrutura deficiente, falta de recursos humanos e humanização no atendimento, retratam a insatisfação do usuário em relação ao sistema de saúde.

Visto isso, a percepção do usuário é de extrema importância ao se dimensionar o reflexo das ações que vêm sendo desenvolvidas no setor saúde, e serve como vetor de direcionamento e planejamento do serviço.

Por fim, afirma-se que o SUS, apesar dos intensos avanços que vem alcançando em seu processo de implementação, ainda tem que promover melhorias na sua organização para que seus princípios sejam efetivos.

\section{Apoio}

Este estudo se desenvolveu no âmbito do Projeto de Inovação em Docência Universitária - PRODUS e foi realizado com o apoio da Coordenação de Aperfeiçoamento de Pessoal de Nível Superior - CAPES, entidade do governo brasileiro voltada para a formação de recursos humanos Processo n 23038.009788/2010-78, AUX-PE-Pró-Ensino Saúde 2034/2010; da 
Fundação de Amparo à Pesquisa do Estado de Minas Gerais - FAPEMIG e do Conselho Nacional de Desenvolvimento Cientifico e Tecnológico - CNPq.

\section{Referências}

1. Martins PC, Cotta RMM, Mendes FF, Priore SE, Franceschini SCC, Cazal MM, et al. De quem é o SUS? Sobre as representações sociais dos usuários do Programa Saúde da Família. Cien Saude Colet. 2011;16(3):1933-12. http://dx.doi.org/10.1590/S1413-81232011000300027.

2. Brasil. Senado Federal. Constituição da República Federativa do Brasil. Brasília (DF): Senado Federal; 1988.

3. Bós AMG, Bós AJG. Determinantes na escolha entre atendimento de saúde privada e pública por idosos. Rev Saude Publica. 2004;38(1):113-20. http://dx.doi.org/10.1590/S003489102004000100016.

4. Stenzel ACB, Misoczky MCA, Oliveira AI. Satisfação dos usuários de serviços públicos de saúde. In: Misoczky MC, Bordin R, organizadores. Gestão local em saúde: práticas e reflexões. Porto Alegre: Dacasa Editora; 2004.

5. Gouveia GC, Souza WV, Luna CF, Szwarcwald CL, Souza-Junior PRB. Satisfação dos usuários com a assistência de saúde no estado de Pernambuco, Brasil, 2005. Cien Saude Colet. 2011;16(3):1849-61. http://dx.doi.org/10.1590/S1413-81232011000300020.

6. Esperidião M, Trad LAB. Avaliação de satisfação de usuários. Cien Saude Colet. 2005;10(Supl1):S303-312. http://dx.doi.org/10.1590/S1413-81232005000500031.

7. Costa GD, Cotta RMM, Ferreira MLS, Reis JR, Franceschini SCC. Saúde da família: desafios no processo de reorientação do modelo assistencial. Rev Bras Enferm. 2009;62(1):113-8. http://dx.doi.org/10.1590/S0034-71672009000100017.

8. Moimaz SAS, Marques JAM, Saliba O, Garbin CAS, Zina LG, Saliba NA. Satisfação e percepção do usuário do SUS sobre o serviço público de saúde. Physis: Rev Saude Colet. 2010;20(4):1419-40. http://dx.doi.org/10.1590/S0103-73312010000400019.

9. Oliveira DF, Arieta CEL, Temporini ER, Kara-José N. Quality of health care: patient satisfaction in a university hospital. Arq Bras Oftalmol. 2006;69(5):731-6. http://dx.doi.org/10.1590/S0004-27492006000500021. 
10. Cotta RMM, Reis RS, Carvalho AL, Batista KCS, Castro FAF, Alfenas RCG. Reflexões sobre o conhecimento dos usuários no contexto do Programa de Saúde da Família: a lacuna entre o saber técnico e o popular. Physis: Rev Saude Colet. 2008;18(4):745-66. http://dx.doi.org/10.1590/S0103-73312008000400008.

11. Yin R. Estudo de caso: planejamento e métodos. 2a ed. Porto Alegre: Bookman; 2001.

12. Bardin L. Análise de Conteúdo. Lisboa: Edições 70; 2008.

13. Milan GS, Trez G. Pesquisa de satisfação: um modelo para planos de saúde. RAE Electron. 2005;4(2). http://dx.doi.org/10.1590/S1676-56482005000200002.

14. Gomes KO, Cotta RMM, Araújo RMA, Cherchiglia ML, Martins TCP. Atenção Primária à Saúde - a "menina dos olhos" do SUS: sobre as representações sociais dos protagonistas do Sistema Único de Saúde. Cien Saude Colet. 2011;16(1):881-92. http://dx.doi.org/10.1590/S1413-81232011000700020.

15. IPEA. Instituto de Pesquisa Econômica Aplicada. SIPS: Sistema de Indicadores de Percepção Social. 2011 [acesso em 2 de jun 2013]. Disponível em: http://www.alames.org/documentos/SIPS.pdf.

16. Ramos DD, Lima MADS. Acesso e acolhimento aos usuários em uma unidade de saúde de Porto Alegre, Rio Grande do Sul, Brasil. Cad Saude Publica. 2003;19(1):27-34. http://dx.doi.org/10.1590/S0102-311X2003000100004.

17. Azevedo ALM, Costa AMA. A estreita porta de entrada do Sistema Único de Saúde (SUS): uma avaliação do acesso na Estratégia de Saúde da Família. Interface. 2010;14(35):797-810. http://dx.doi.org/10.1590/S1414-32832010005000029.

18. Brasil. Ministério da Saúde (MS). Secretaria de Políticas de Saúde. Departamento de Atenção Básica. Política Nacional de Medicamentos 2001. Brasília (DF): Ministério da Saúde; 2001.

19. Souza JJG. Hipertensão arterial referida e uso de anti-hipertensivos em adultos na cidade de São Paulo, 2003: um estudo de base populacional [dissertação]. [São Paulo]: Universidade de São Paulo; 2006. 112 f.

20. Boing AC, Bertoldi AD, Boing AF, Bastos JL, Peres KG. Acesso a medicamentos no setor público: análise de usuários do Sistema Único de Saúde no Brasil. Cad. Saude Publica. 2013;29(4):691-701. http://dx.doi.org/10.1590/S0102-311X2013000400007.

21. Viegas SMF, Penna CMM. O SUS é universal, mas vivemos de cotas. Cien Saude Colet. 
2013;18(1):181-90. http://dx.doi.org/10.1590/S1413-81232013000100019.

22. De Lavor A, Dominguez B, Machado K. O SUS que não se vê. RADIS. 2011 abr [acesso em 10 jun 2013];(104). Disponível em: http://www.ensp.fiocruz.br/radis/revistaradis/104/reportagens/o-sus-que-nao-se-ve.

23. Chernicharo IM, Freitas FDS, Ferreira MA. Humanização no cuidado de enfermagem: contribuição ao debate sobre a Política Nacional de Humanização. Rev Bras Enferm. 2013;66(4):564-70. http://dx.doi.org/10.1590/S0034-71672013000400015.

24. Brasil. Ministério da Saúde (MS). Núcleo Técnico da Política Nacional de Humanização. Humaniza SUS. Política nacional de humanização: a humanização como eixo norteador das práticas de atenção e gestão em todas as instâncias do SUS. Brasília (DF): Ministério da Saúde, 2004.

25. Cotta RMM, Reis RS, Campos AAO, Gomes AP, Antonio VE, Siqueira-Batista R. Debates atuais em humanização e saúde: quem somos nós? Cien Saude Colet. 2013;18(1):171-9. http://dx.doi.org/10.1590/S1413-81232013000100018.

26. Cotta RMM, Mendonça ET, Costa GD. Portfólios reflexivos: construindo competências para o trabalho no Sistema Único de Saúde. Rev Panam Salud Publica. 2011;30(5):415-21. 


\section{Minicurrículo}

Rosângela Minardi Mitre Cotta | ORCiD: 0000-000

Professora Titular da Universidade Federal de Viçosa - UFV, Departamento de Nutrição e Saúde - DNS.

Doutora em Saúde Coletiva pela Universidad de Valencia, Espanha. Coordenadora do Laboratório de Estudos em Planejamento e Gestão em Saúde - LABPlanGest e do Programa de Inovação em Docência Universitária PRODUS, da UFV.

Aline Aparecida de Oliveira Campos | ORCiD: 0000-0002-3795-2588

Professora Adjunto A da Universidade Federal do Sul e Sudeste do Pará - Unifesspa, Instituto de Estudos em Saúde e Biológicas - IESB, Faculdade de Saúde Coletiva - FASC. Doutora e Mestre em Ciência da Nutrição pela Universidade Federal de Viçosa - UFV.

Luciana Saraiva da Silva | ORCiD: 0000-0001-9059-4399

Professora Adjunta da Universidade Federal de Uberlândia - UFU, Faculdade de Medicina, Curso de Nutrição. Doutora e Mestre em Ciência da Nutrição pela Universidade Federal de Viçosa - UFV.

\section{Lucas Saraiva da Silva}

Médico pela Universidade Federal Fluminense - UFF.

\section{Fernanda Mitre Cotta}

Médica Ginecologista e Obstetra. Residente de Mastologia no Hospital das Clínicas da Universidade Federal de Minas Gerais - UFMG.

\section{Rodrigo Mitre Cotta}

Médico Residente em Ortopedia e Traumatologia pela Santa Casa de Misericórdia de Belo Horizonte.

\section{Lia Márcia Cruz da Silveira}

Psicóloga. Mestre em Tecnologia Educacional nas Ciências da Saúde - NUTES/UFRJ. Especialista em Psicologia Médica - UERJ e em Ativação de Processos de Mudança da Formação Superior de Profissionais de Saúde - ENSP /FIOCRUZ. Técnica em Assuntos Educacionais HESFA/UFRJ. Coordenadora Pedagógica do Projeto Preceptoria ABEM original (2012-2016).

Denise Herdy Afonso | ORCiD: 0000-0002-4781-0364

Professora do Departamento de Medicina Integral, Familiar e Comunitária. Faculdade de Ciências Médicas FCM. Coordenadora do Grupo Com Vida, Hospital Universitário Pedro Ernesto - HUPE. Universidade do Estado do Rio de Janeiro - UERJ. Coordenadora do Projeto Preceptoria ABEM original (2012-2016). Especialista em Educação de Profissionais de Saúde - UF Ceará - FAIMER 2010. Mestre em Ciências Médicas Educação e Informação - FCM - UERJ. 